\title{
Sensitivity Analysis of a Bidirectional Wireless Charger for EV
}

\author{
Alicia Triviño Cabrera, José A. Aguado Sánchez \\ Dpto. Ingeniería Eléctrica \\ University of Málaga \\ Málaga, Spain \\ atc,jaguado@uma.es
}

\author{
Michela Longo, Federica Foiadelli \\ Dept. of Energy \\ Politecnico di Milano \\ Milan, Italy \\ michela.longo@polimi.it
}

\begin{abstract}
Bidirectional chargers are required to fully integrate Electric Vehicle (EV) into the smart grids. Additionally, wireless chargers ease the charge/discharge process of the EV batteries so that they are becoming more popular to fulfill a V2G scenario. When considering the load of wireless chargers, it is a requirement to know the real output power that these systems offer. The designed output power may differ from the real one as components suffer from tolerance. This paper defines six sensitivity factors to model the severity of the effects of tolerance into the output power. To do so, an electric circuit analysis is used and a mathematical formulation is derived. The six sensitivity factors are computed for a laboratory prototype.
\end{abstract}

Keywords-component; tolerance, sensitivity, Electric Vehicle, wireless charger, bidirectional

\section{INTRODUCTION}

Smart grids must cope with the impact of new loads and, in particular, with Electric Vehicles (EV) [1,2]. Electric vehicles will play a fundamental role in a future V2G (Vehicle-to-Grid) scenario as it may act as a consumer, when being charged, and as a producer, when it delivers energy to the electrical network $[3,4]$. This double functionality is expected to enhance the grid efficiency as it allows to flatten the demand curves [5]. Specific agents are incorporated in order to support the way the EVs interact with the grid.

Electric Vehicles can be charged/discharged by a conductive or a wireless based technology [6-8]. The traditional conductive approach relies on the physical connection between the charger and the EV. As an alternative, wireless chargers are becoming more popular as they reduce the user's intervention during the charge process and it also allows to recharge the vehicle even when it is moving $[9,10]$. Wireless and conductive chargers must be bidirectional if we aim to work with $\mathrm{EV}$ in a V2G scenario, that is, that the EV battery receives/delivers energy from/to the grid [11]

In order to assume correct EV loads when planning smart grids, a precise understanding on the way EV chargers work and how efficient they are required [12]. This comprehension should consider the realistic behavior of wireless chargers, which are affected by the tolerance of their components. Tolerance makes the nominal of the discreet elements vary from the design parameters, which is a feasible consequence in high volume manufacturing. By means of simulations, the work in [13] analyses the effects of the components' tolerance in the behavior of unidirectional wireless chargers.

In contrast to the previous work, this paper focuses on bidirectional wireless chargers and it follows a mathematical approach. Specifically, we study the power delivered to the battery by real wireless chargers when the tolerance of the EV components is considered. In this sense, we formulate a sensitivity analysis of a Series-Series bidirectional wireless charger. The mathematical formulation provides with six sensitivity factors. From this study, we conclude by which components' tolerance, the power delivered to the battery is affected more. This study gives some insights about which components should be selected carefully in order to get the desired output power and, as a consequence, the expected load. The conclusions may help designers to adapt control schemes in the wireless chargers to reduce the effects of tolerances in a similar way to the approach in [14], which is intended for conducive charger. As an illustrative application of our derived study, we have applied the sensitivity analysis to a $3.7 \mathrm{~kW}$ wireless charger with a resonant frequency equal to $85 \mathrm{kHz}$.

The remainder of the paper is structured as follows. Section II explains the basics of a resonant wireless charger for EV. Section III includes the sensitivity analysis. The application of this study into a designed prototype is presented in Section IV. Finally, Section V draws the main conclusions of the paper.

\section{WIRELESS CHARGER FOR AN EV}

The core of a resonant wireless charger is two coupled coils, named transmitter and receiver. Both coils are adapted with reactive structures so that the whole system is on resonance conditions at a specific designed frequency. In this way, the battery gets the maximum real power from the grid.

Depending on the components of the reactive structures, the compensation topologies can be classified into single-resonant and multi-resonant [15]. The single-resonant structures adds a capacitor to each coil whereas multi-resonant topologies use multiple reactive components in the transmitter and/or the receiver coil.

Due to their robustness, single-resonant compensation topologies are frequently used. In this category, there are four compensation topologies: Series - Series (SS), Series - Parallel (SP), Parallel - Series (PS) and Parallel - Parallel (PP). The first word stands for the connection between the primary capacitor 
and the transmitter coil while the second word refers to the type of connection between the secondary capacitor and the receiver coil. When working with bidirectional chargers, it is preferred to opt for symmetric compensation topologies that ease the control implementation, that is, the procedures that generate the signals to tune complementary power converters to work in one sense (energy flowing from the grid to the battery) or another (energy flowing from the battery to the grid). SS compensation topology presents an additional advantage: the design of the capacitors are independent in the primary and in the secondary side. That means that the designed values for these two reactive components are exclusively derived from their corresponding coils and it is not necessary to take into account other components, which will make the design process more complicated. Figure 1 shows the core of a resonant wireless charger with a SS compensation topology. The sinusoidal source is derived from a power converter which is able to transform the electrical input frequency $(50-60 \mathrm{~Hz})$ into a higher frequency $(85$ $\mathrm{kHz}$ in our study) and vice versa. The load is modeled as a resistance (RL) as it imposes a constant voltage and it demands a pre-defined real power.

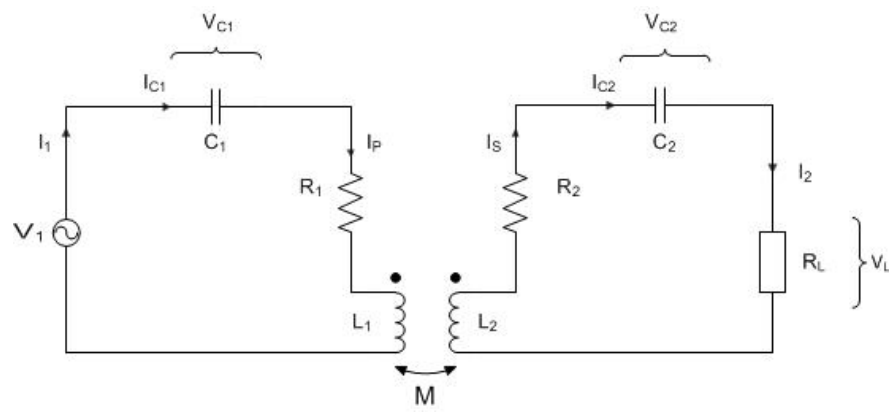

Fig. 1. SS compensation topology for an EV wireless charger

The design process of a SS-based wireless charger starts by setting an operational frequency at which the coils will be resonant. The angular operational frequency is $\omega_{o}$. Then, we should select the coils geometry and their material. Their inductance (which is based on the number of turns in the coil) and the associated capacitors are set taking into account some design guidelines such as avoiding bifurcation, supporting a specific current density or using a reduced amount of material. Multiple potential solutions are obtained in this step. In all of them, there is a relationship between the inductance and the capacitor of both sides. To guarantee the resonance operation, the values in the primary and in the secondary resonant tanks are related as follows:

$$
\begin{aligned}
& \omega_{0}=\frac{1}{\sqrt{L_{1} \cdot C_{1}}} \\
& \omega_{0}=\frac{1}{\sqrt{L_{2} \cdot C_{2}}}
\end{aligned}
$$

where $L_{1}$ stands for the auto-inductance of the primary coil, $L_{2}$ for the auto-inductance of the secondary coil, $C_{1}$ is the capacitor of the primary side and $C_{2}$ is the capacitor in the secondary side.
The goodness of each potential solution may be evaluated following a heuristic, which will help the designer to identify the final configuration in the solution space.

Assuming a first harmonic approximation, the electrical analysis of the resonant wireless structures is as follows. Firstly, we identify three impedances: the secondary impedance $Z_{2}$, the primary impedance $Z_{1}$ and the total primary impedance $Z_{1 T} . Z_{1}$ and $Z_{2}$ are computed assuming no coupling between the transmitter and the receiver. Then, $Z_{1 T}$ represents the impedance seen by the source when coupling happens, that is, when $Z_{2}$ is reflected into the primary side. The equations for these impedances are the following ones:

$$
\begin{gathered}
Z_{1}=R_{1}+j\left(\omega_{o} L_{1}-\frac{1}{\omega_{0} C_{1}}\right) \\
Z_{2}=R_{L}+R_{2}+j\left(\omega_{o} L_{2}-\frac{1}{\omega_{0} C_{2}}\right) \\
Z_{1 T}=Z_{1}+\frac{\left(\omega_{0} M\right)^{2}}{Z_{2}}
\end{gathered}
$$

The coupling effect in the secondary side can be modelled as an induced voltage. The value of the voltage is $j \omega_{o} M \overline{I_{1}}$. So, once the impedances are computed, we proceed to derive the currents in the primary side $\left(\overline{I_{1}}\right)$ and in the secondary side $\left(\overline{I_{2}}\right)$.

$$
\begin{gathered}
\overline{I_{1}}=\frac{V_{1}}{Z_{1 T}} \\
\overline{I_{2}}=\frac{j \omega_{0} M \overline{I_{1}}}{Z_{2}}
\end{gathered}
$$

The real output power delivered to the load $\left(P_{L}\right)$ is:

$$
P_{L}=R_{L} \cdot I_{2}^{2}
$$

\section{SENSITIVITY ANALYSIS}

Real components suffer from deviations in their nominal values, effect which is known as tolerance. A sensitivity analysis let us know how the tolerance impacts on the output power. In the present work, the tolerances considered are the one associate with the primary coil's inductance and resistance, with the primary capacitor, with the secondary coil's inductance and resistance and with the secondary capacitor. We derive six sensitivity factors related to the output power: sensitivity to the primary capacitance $\left(S_{C_{1}}\right)$, sensitivity to the secondary capacitance $\left(S_{C_{2}}\right)$, sensitivity to the primary coil's inductance $\left(S_{L_{1}}\right)$, sensitivity to the primary coil's resistance $\left(S_{R_{1}}\right)$, sensitivity to the secondary coil's inductance $\left(S_{L_{2}}\right)$ and sensitivity to the secondary coil's resistance $\left(S_{R_{2}}\right)$.

The sensitivity of the output power to deviations in the primary capacitance $\left(S_{C_{1}}\right)$ is expressed in Equation 10 and 11. For this formulation, we assume that there is one variation occurring in only one component of the wireless charger. 


$$
S_{C_{1}}=\frac{d P_{L}}{d C_{1}}=2 R_{L} I_{2} \frac{d I_{2}}{d C_{1}}
$$

By using Equations 7 and 8, we can further develop Eq. 10 leading to Eq. 11:

$$
S_{C_{1}}=2 R_{L} I_{2} \frac{\omega_{0} M}{Z_{2}} \frac{d I 1}{d C_{1}}=2 R_{L} I_{2} \frac{\omega_{0} M}{Z_{2}} \frac{V_{1}}{Z_{1 T}^{2} \omega_{0} C_{1}^{2}}
$$

In a similar way, we can specify $S_{C_{2}}$ as shown in Eq. 12:

$$
S_{C_{2}}=\frac{d P_{L}}{d C_{2}}=2 R_{L} I_{2} \frac{d I_{2}}{d C_{2}}
$$

We can extend this expression by considering Equations 8 and 5. As a result, we get the following sensitivity factor:

$$
S_{C_{2}}=2 R_{L} I_{2} \omega_{0} M\left(\frac{d I_{1}}{d C_{2}} \frac{1}{Z_{2}}+I_{1} \frac{d 1 / Z_{2}}{d C_{2}}\right)
$$

where

$$
\begin{gathered}
\frac{d I_{1}}{d C_{2}}=\frac{V_{1} \omega_{0} M^{2}}{Z_{1 T}^{2} Z_{2}^{2} C_{2}^{2}} \\
\frac{d 1 / Z_{2}}{d C_{2}}=\frac{-1}{Z_{2}^{2} C_{2}^{2} \omega_{0}}
\end{gathered}
$$

Alternatively, the sensitivity factors $S_{L_{1}}$ and $S_{L_{2}}$ are derived from the previous sensitivity factors and the resonant conditions defined in Equations 1 and 2.

$$
\begin{gathered}
S_{L_{1}}=\frac{d P_{L}}{d L_{1}}=\frac{d P_{L}}{d C_{1}} \frac{d C_{1}}{d L_{1}}=\frac{-S_{C_{1}}}{\omega_{0}^{2} L_{1}^{2}} \\
S_{L_{2}}=\frac{d P_{L}}{d L_{2}}=\frac{d P_{L}}{d C_{2}} \frac{d C_{2}}{d L_{2}}=\frac{-S_{C_{2}}}{\omega_{0}^{2} L_{2}^{2}}
\end{gathered}
$$

Finally, we obtain the sensitivity of the output power to the coils internal resistances. For the primary coil, this parameter is computed as follows:

$$
S_{R_{1}}=\frac{d P_{L}}{d R_{1}}=2 R_{L} I_{2} \frac{d I_{2}}{d R_{1}}=\frac{-2 R_{L} I_{2} \omega M V_{1}}{Z_{2} \cdot Z_{1 T}^{2}}
$$

On the other hand, the sensitivity of the output power of the bidirectional wireless charger to the internal resistance of the secondary coil is expressed as follows:

$$
S_{R_{2}}=\frac{d P_{L}}{d R_{2}}=2 R_{L} I_{2} \omega M\left(\frac{d I_{1}}{d R_{2}} \frac{1}{Z_{2}}+I_{1} \frac{d 1 / Z_{2}}{d R_{2}}\right)
$$

where

$$
\begin{gathered}
\frac{d I_{1}}{d R_{2}}=\frac{V_{1} \omega_{0}^{2} M^{2}}{Z_{1 T}^{2} Z_{2}^{2}} \\
\frac{d 1 / Z_{2}}{d R_{2}}=\frac{-1}{Z_{2}^{2}}
\end{gathered}
$$

\section{APPLICATION TO A PROTOTYPE}

In this Section, we compute the sensitivity factors for a real wireless charger prototype. In Table I, the values of the components adopted for the final prototype are shown. For the design process, the following specifications are considered: 3.7$\mathrm{kW}$ output power, $300-\mathrm{V}$ output voltage specifications and 85$\mathrm{kHz}$ resonance frequency. We have opted for rectangular coils built with Litz cable. The primary coil has $N_{1}$ turns of $\mathrm{a}_{1} \mathrm{xb}_{1} \mathrm{~m}^{2}$ whereas the secondary coil is composed of $N_{2}$ turns of $\mathrm{a}_{2} \mathrm{xb}_{2} \mathrm{~m}^{2}$. The coil diameter for both structures is $s$. There is a distance between the two coils equal to $20 \mathrm{~cm}$.

TABLE I. EV CHARGER SPECIFICATIONS, DESIGN VALUES OF THE COMPONENTS

\begin{tabular}{|c|c|c|c|}
\hline \multicolumn{2}{|c|}{ Charger specifications } & \multicolumn{2}{c|}{$\begin{array}{c}\text { TX-RX parameters } \\
\text { (design values) }\end{array}$} \\
\hline Output & 3.7kW@300V & $\mathrm{L}_{1}[\mu \mathrm{H}]$ & 271.0 \\
\hline $\mathrm{fs}[\mathrm{kHz}]$ & 85 & $\mathrm{~L}_{2}[\mu \mathrm{H}]$ & 252.0 \\
\hline \multicolumn{2}{|c|}{ Coils geometry } & $\mathrm{C}_{1}[\mathrm{nF}]$ & 12.9 \\
\hline $\mathrm{N}_{1}$ & 11 & $\mathrm{C}_{2}[\mathrm{nF}]$ & 13.9 \\
\hline $\mathrm{N}_{2}$ & 14 & $\mathrm{R}_{1}[\mathrm{~m} \Omega]$ & 30.9 \\
\hline $\mathrm{s}\left[\mathrm{mm}^{2}\right]$ & 20 & $\mathrm{R}_{2}[\mathrm{~m} \Omega]$ & 27.8 \\
\hline $\mathrm{a}_{1} \mathrm{xb}_{1}\left[\mathrm{~m}^{2}\right]$ & $0.75 \times 0.75$ & $\mathrm{M}[\mu \mathrm{H}]$ & 40.8 \\
\hline $\mathrm{a}_{2} \times \mathrm{b}_{2}\left[\mathrm{~m}^{2}\right]$ & $0.5 \times 0.5$ & & \\
\hline $\mathrm{h}[\mathrm{m}]$ & 0.2 & & \\
\hline
\end{tabular}

Using the previous equations, we get the sensitivity factors for this particular prototype. The obtained results are summarized in Table II.

TABLE II. OUTPUT POWER SENSITIVITY FACTORS

\begin{tabular}{|c|c|}
\hline \multicolumn{2}{|c|}{ Output power sensitivity factors } \\
\hline$S_{C_{1}}[\mathrm{~W} / \mathrm{nF}]$ & $8.5 \cdot 10^{3}$ \\
\hline$S_{C_{2}}[\mathrm{~W} / \mathrm{nF}]$ & $-27.8 \cdot 10^{3}$ \\
\hline$S_{L_{1}}[\mathrm{~W} / \mu \mathrm{H}]$ & 166 \\
\hline$S_{L_{2}}[\mathrm{~W} / \mu \mathrm{H}]$ & 3750 \\
\hline$S_{R_{1}}[\mathrm{~W} / \mathrm{m} \Omega]$ & $-3.10 \cdot 10^{5}$ \\
\hline$S_{R_{2}}[\mathrm{~W} / \mathrm{m} \Omega]$ & 587 \\
\hline
\end{tabular}

As can be observed, the tolerance of the reactive components placed in the secondary side have a higher impact than the deviations of the values of the primary reactive elements. However, if we focus on the coils' internal resistance, we can conclude that the variations of the primary resistance is more relevant than those occurring in the secondary coil. 
As a future guideline, the sensitivity factors may be considered in the design process so that we opt for the configuration with limited sensitivity factors among the solution space.

\section{CONCLUSIONS}

Wireless chargers are foreseen as the key to promote the use of electric vehicles in the smart grid. The basic of this kind of system relies on two coupled coils with compensation systems to maximize the power transferred to/from the battery. This paper formulates a mathematical study about the sensitivity of the output power delivered by a bidirectional Series-Series system to the variations of the components' nominal value. Six sensitivity factors are analytically obtained. Their values are also obtained in a prototype of an EV bidirectional wireless charger.

The results show that the deviations of the values associated to the reactive components of the secondary tank greatly impact on the system performance. In contrast, the internal resistance of the primary coil should be carefully computed in the design process as the deviations of this value lead to more severe consequences than those due to the internal resistance of the secondary coil.

\section{ACKNOWLEGMENTS}

This work has been partially funded by Universidad de Málaga, Campus de Excelencia Internacional Andalucia Tech.

\section{REFERENCES}

[1] B. Gadalla, E. Schaltz, F. Blaabjerg, "A survey on the reliability of power electronics in electro-mobility applications", Joint International Conference - ACEMP 2015: Aegean Conference on Electrical Machines and Power Electronics, OPTIM 2015: Optimization of Electrical and Electronic Equipment and ELECTROMOTION 2015: International Symposium on Advanced Electromechanical Motion Systems.

[2] A.K. Baghel, P. Kumar, P. Kumar, "Scenario of electric and hybrid electric vehicles by 2030", 2015 IEEE International Transportation Electrification Conference, ITEC-India 2015.

[3] F. Mwasilu, J. Justo, E-K Kim, T. Do, J-W Jung, "Electric vehicles and smart grid interaction: A review on vehicle to grid and renewable energy sources integration", Renewable and Sustainable Energy Reviews, vol. 34, pp. 501-516, June 2014.

[4] M. Brenna, F. Foiadelli, M. Longo, "The exploitation of vehicle-to-grid function for power quality improvement in a smart grid", IEEE Transactions on Intelligent Transportation Systems, vol. 15, Issue 4, pp. 2169-2177, 2014.

[5] P. Dost, F. Einwächter, P. Spichartz, C. Sourkounis, "Influence of electric vehicle charging demands on the grid load based on fleet measurements", IEEE Vehicle Power and Propulsion Conference, VPPC 2014.

[6] E. van Wynsberghe, A. Turak, "Station-keeping of a high-altitude balloon with electric propulsion and wireless power transmission: A concept study", Acta Astronautica, 128, pp. 616-627, 2016.

[7] L. Bedogni, L. Bononi, M. Di Felice, A. D'Elia, R. Mock, F. Morandi, S. Rondelli, T. Salmon Cinotti, F. Vergari, "An Integrated Simulation Framework to Model Electric Vehicle Operations and Services", IEEE Transactions on Vehicular Technology, 65 (8), pp. 5900-5917, 2016.

[8] M. Kabalo, F. Berthold, B. Blunier, D. Bouquain, S. Williamson, A. Miraoni, "Efficiency comparison of wire and wireless battery charging: Based on connection probability analysis", 2014 IEEE Transportation Electrification Conference and Expo: Components, Systems, and Power Electronics - From Technology to Business and Public Policy, ITEC 2014.

[9] L. Jian, H. Xue, G. Xu, X. Zhu, D. Zhao, and Z. Y. Shao, "Regulated charging of plug-in hybrid electric vehicles for minimizing load variance in household smart microgrid," IEEE Trans. Ind. Electron., vol. 60, no. 8, pp. 3128-3226, Aug. 2013.

[10] F. Musavi, M. Edington, and W. Eberle, "Wireless power transfer: survey of EV battery charging technologies," in Proc. Energy Convers. Congr. Expo., 2012, pp. 1804-1801.

[11] J-Y. Lee, B-M. Han, "A Bidirectional Wireless Power Transfer EV Charger Using Self-Resonant PWM", IEEE Transactions on Power Electronics, vol. 30, Iss. 4, pp. 1784 - 1787, 2015.

[12] S. Raghavan, "Impact of demand response on Electric Vehicle charging and day ahead market operations", Proc. IEEE Power and Energy Conference, pp. 1-7, 2016

[13] A. Triviño, D. Fernández, J. A. Aguado, J. E. Ruiz, "Sensitivity Analysis of Component's Tolerance in Inductively Coupled Power Transfer System", Proceedings of 2013 International Conference on Renewable Energy Research and Applications, ICRERA 2013.

[14] J. Park. M. Kim, S. Choi, "Zero-current switching series-loaded resonant converter insensitivie to resonant component tolerance for battery charger", IET Power Electronics, vol. 4, Iss. 7, pp. 2517-2524, 2014.

[15] U. K. Madawala, M. Neath, D. J. Thrimawithana, "A Power-Frequency controller for bidirectional inductive power transfer systems", IEEE Trans. Ind. Electron., vol. 60, n⿳⺈ 1, pp. 310-317, January 2013. 